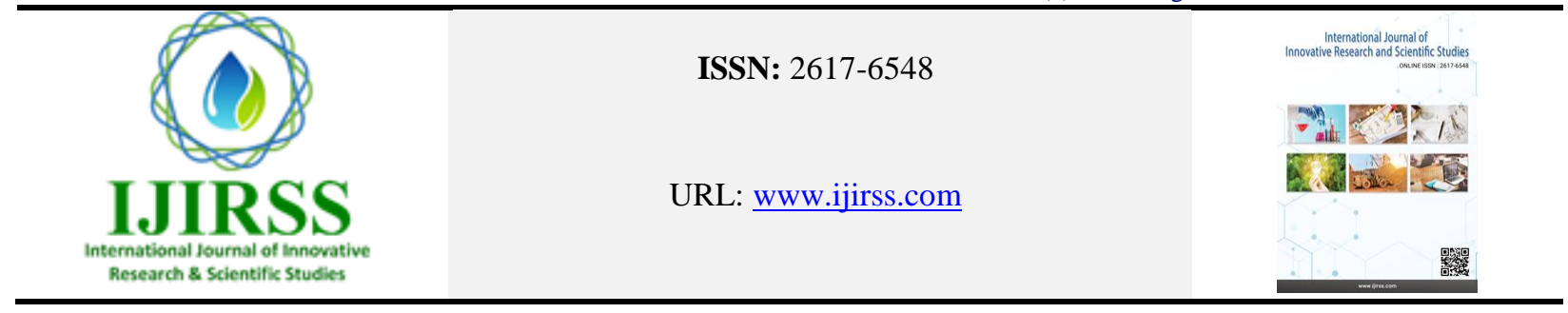

\title{
Evaluation of Technical Drawing Rules and its Application in Engineering Drawings and Final Year Projects
}

\author{
Mohammad Maruf Sharifi \\ Faculty of Construction Engineering, Jawzjan University, Jawzjan, Afghanistan \\ (Email: maruf.sharifi123@gmail.com)
}

\begin{abstract}
The way of imagination and visualization of spatial, the ability of read, analyze and interpret different drawings for engineering students is provided by graphics training. The accurate way of technical drawings and rules in engineering drawing in final year projects are discussed in this paper. Primary and main material collection was done by distribution of questionnaires amongst the final year students and also by collecting their look outs based on a survey questionnaire amongst 300 students from different engineering departments. 300 different final year projects and 2500 engineering drawings were surveyed from a batch of students from 2016-2018. Although, the design drawings and architecture drawings in civil and architecture departments take around 90 sheets, whereas simple drawings are sparse. But in the field of geology and mind, oil and gas, hydraulics have majority of infrastructure and simpler drawings. Conclusively, the application of technical drawings is same in all departments, while in civil and Architecture department's projects, scales, thicknesses of lines, types of lines are used correctly and due to wrong use of symbols and colors is rejected. In Geology and mind, oil and gas and hydraulic due to the use of large scales, colors are accepted but, on basis of incorrect use of line thicknesses and small dimensions are rejected.
\end{abstract}

Keywords: Technical drawings, Projects, Engineering drawings.

DOI: $10.53894 /$ ijirss.v3i3.38

Funding: This study received no specific financial support.

History: Received: 8 June 2020/Revised: 6 July 2020/Accepted: 27 July 2020/Published: 3 August 2020

Licensed: This work is licensed under a Creative Commons Attribution 4.0 License (cc) EY

Competing Interests: The author declares that there are no conflicts of interests regarding the publication of this paper.

Transparency: The author confirms that the manuscript is an honest, accurate, and transparent account of the study was reported; that no vital features of the study have been omitted; and that any discrepancies from the study as planned have been explained.

Ethical: This study follows all ethical practices during writing. 


\title{
بررسى قوانين رسم تخنيك و كاربرد آن در نقشه هاى انجنيرى و بِروزه هاى دفاع دييلوم
}

\author{
محمد معروف شريفى \\ دانثكده انجنيرى ساختمانى، دانثكاه جوزجان، جوزجان، افغاستان
}

\begin{abstract}
خلاصه

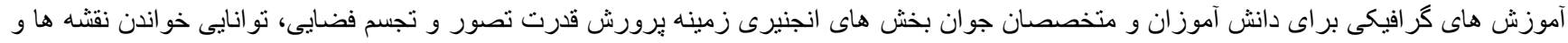

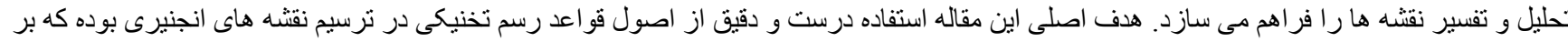

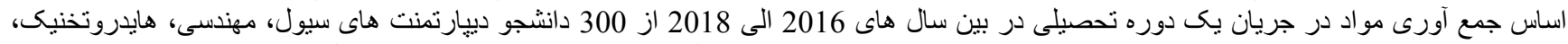

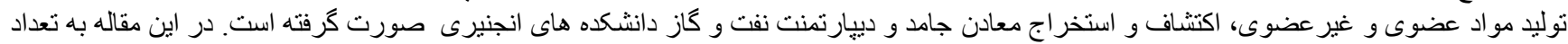

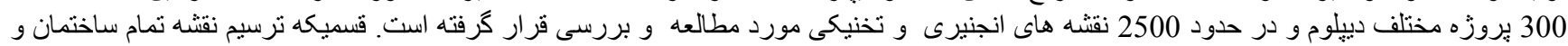

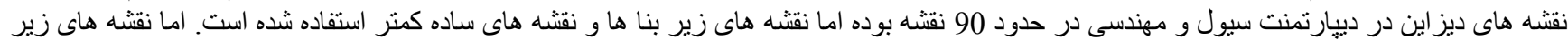

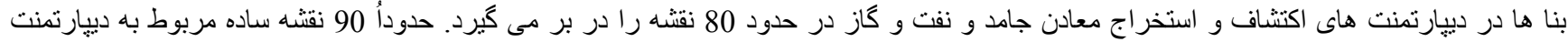

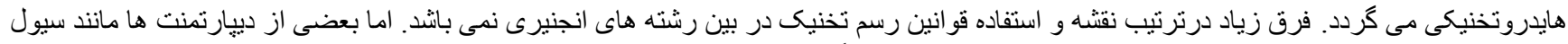

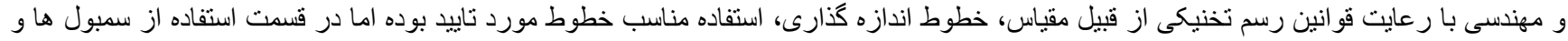

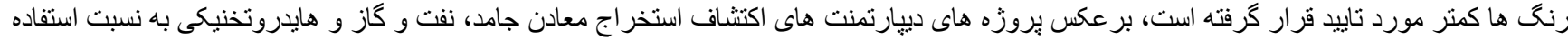

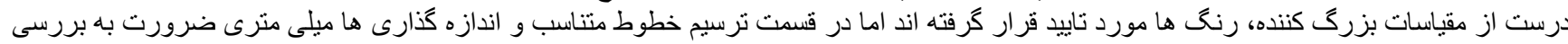
مجدد مى داشته باثند.

كلمات كليدى: رسم تخنيك، بروزه ها، نقشه هاى انجنيرى

1 1

.1

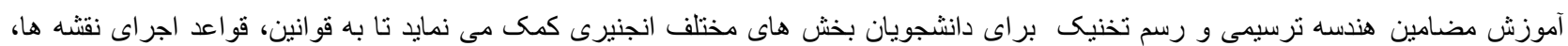

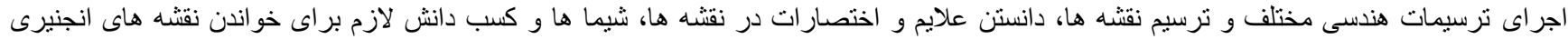

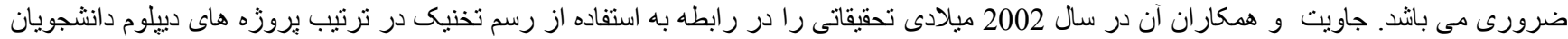

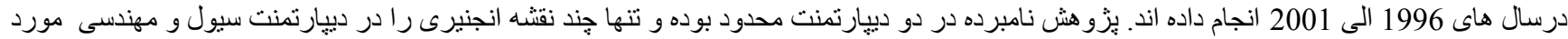

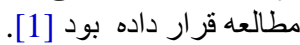

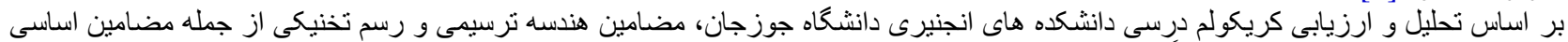

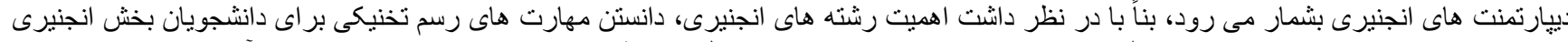

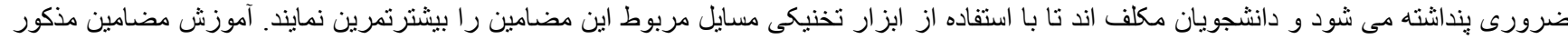

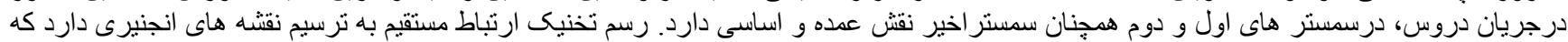

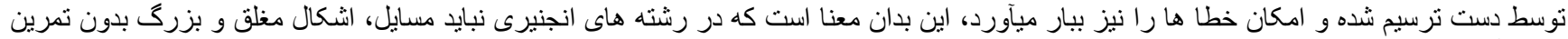

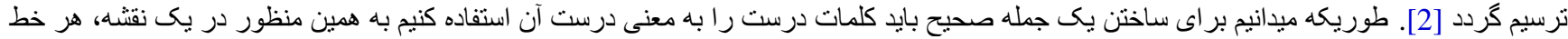

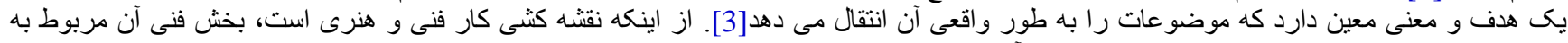

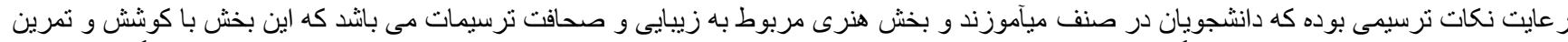

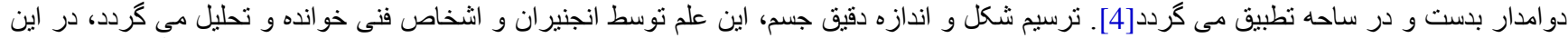

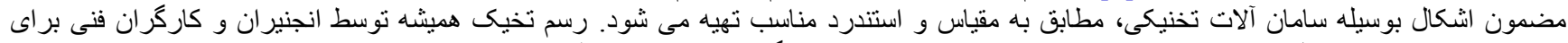

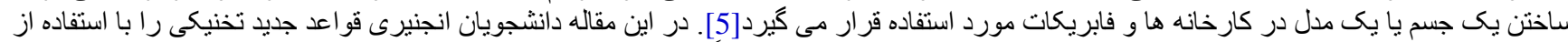

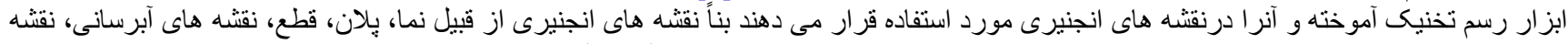

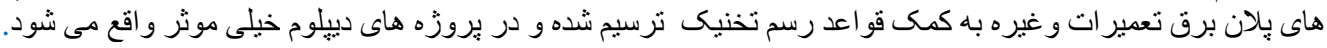

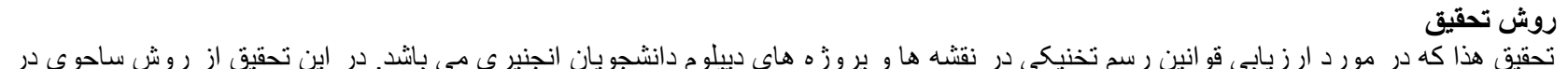

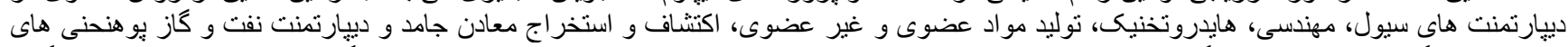

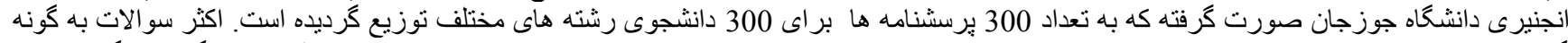

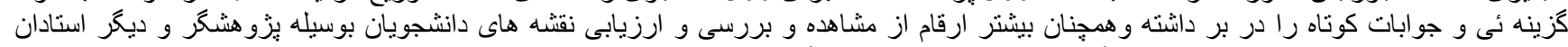

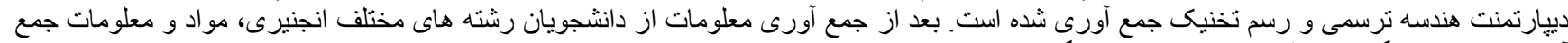

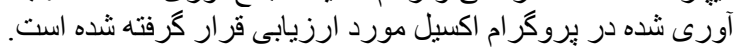

1.2

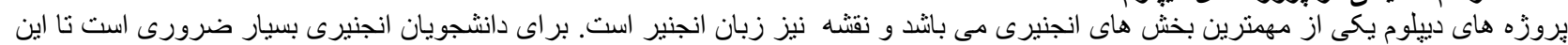

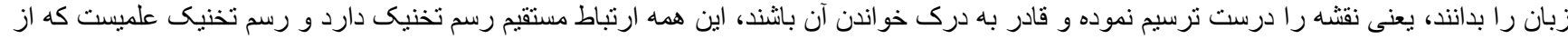

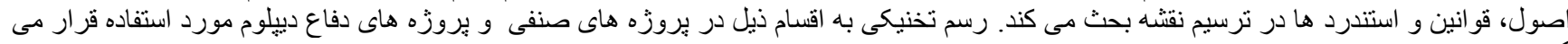
رسم هندسى: نمايش اشكال هندسى مانند مربع، مستطيل، دايره، مثلث، كثير الاضلاع، استو انه، مخروط، كره، هرم و غيره رسم هندسى ناميده مى شود. رسم

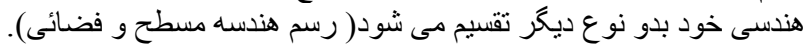




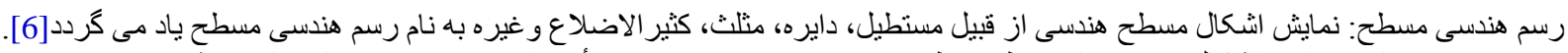

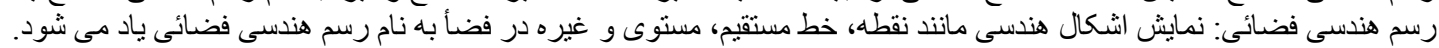

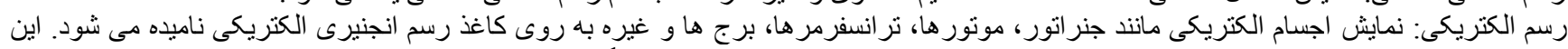

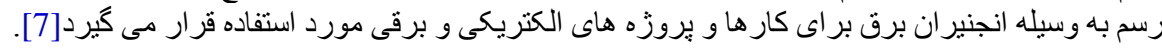

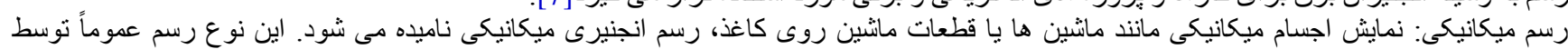

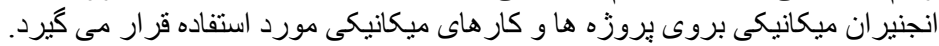

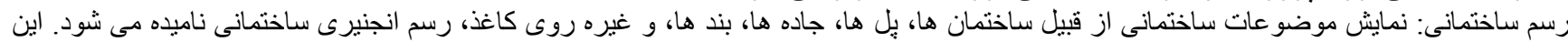

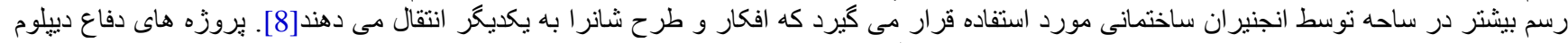

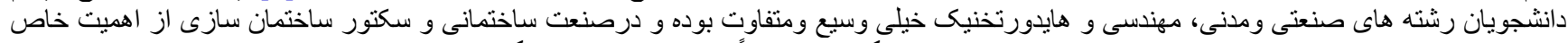

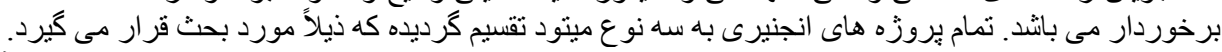

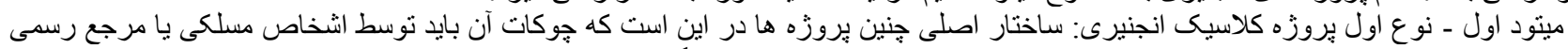

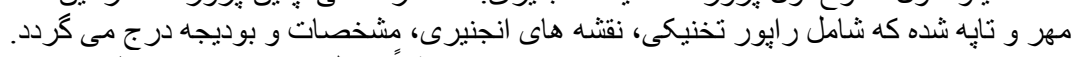

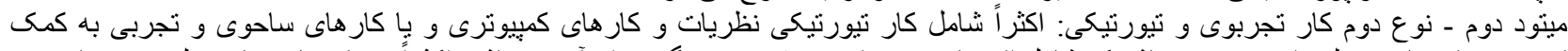

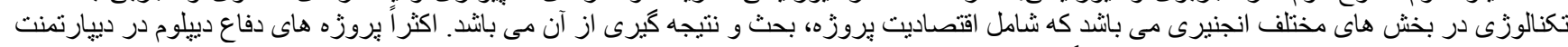

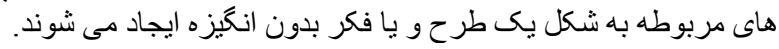

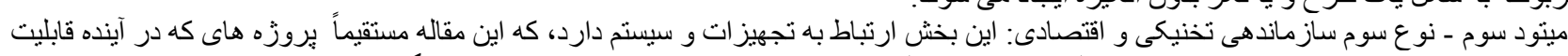

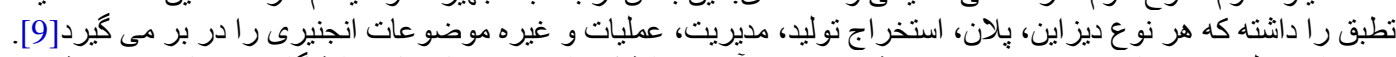

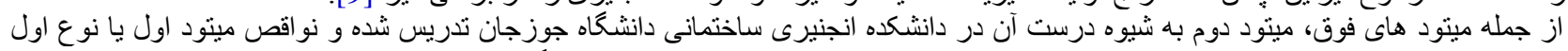

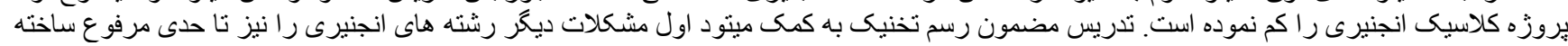

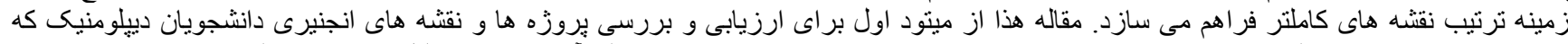

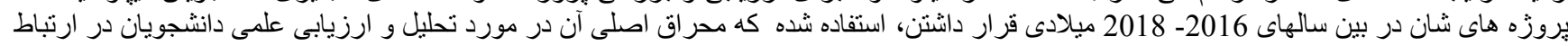

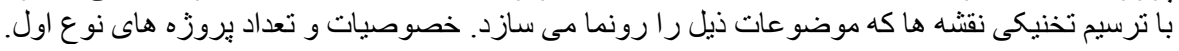

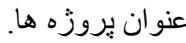

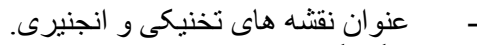

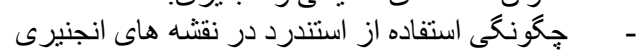

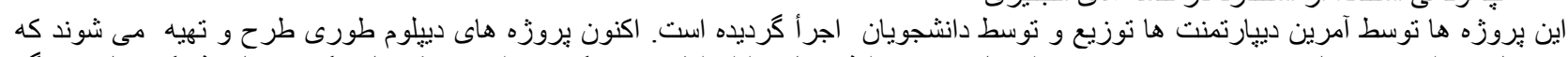

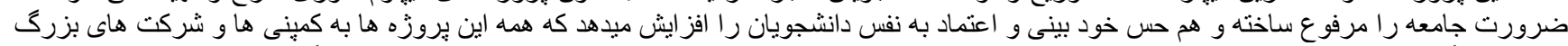

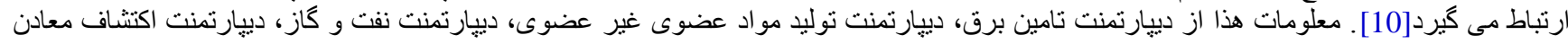

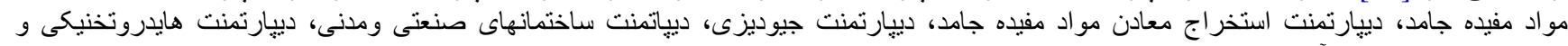

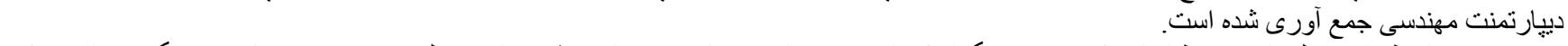

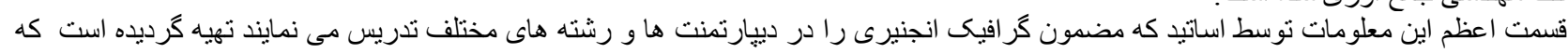

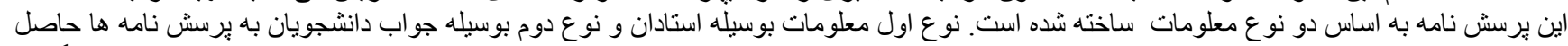

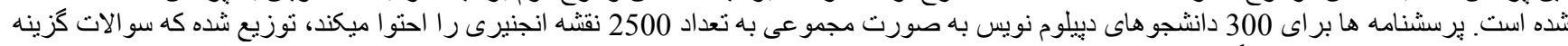

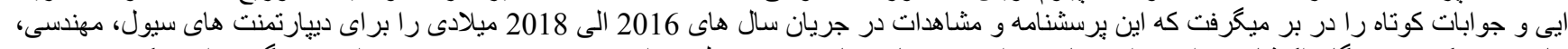

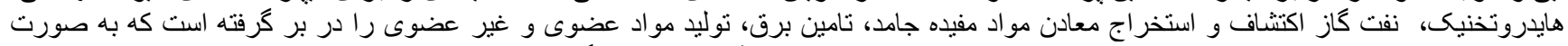

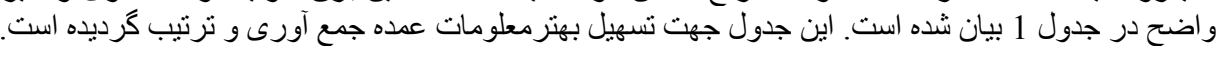

\begin{tabular}{|c|c|c|c|}
\hline تيروز هاده & سال ها & رشته هاى تخصصى & هجموعى نقشنه \\
\hline 300 & $\begin{array}{l}2016 \\
2017 \\
2018\end{array}$ & 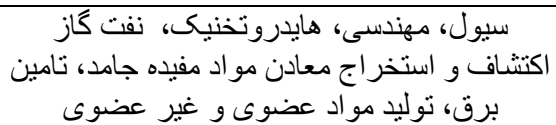 & 2500 \\
\hline
\end{tabular}

1.3

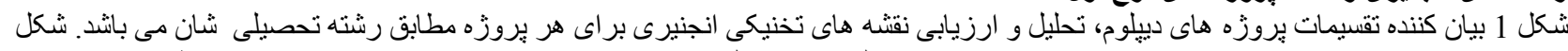

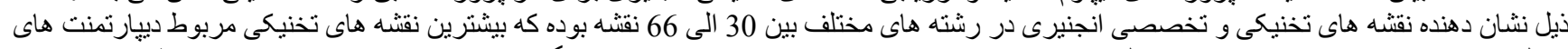

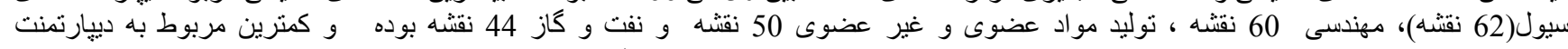

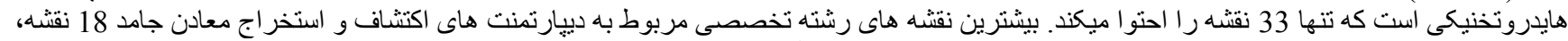

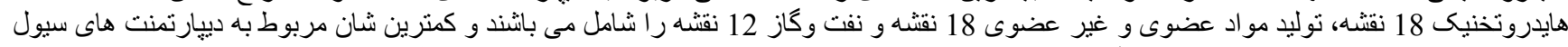

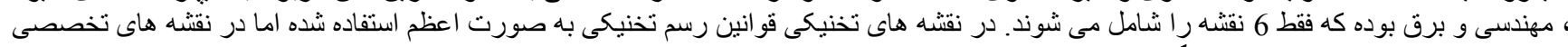

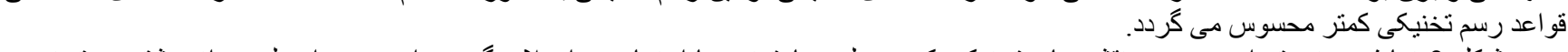

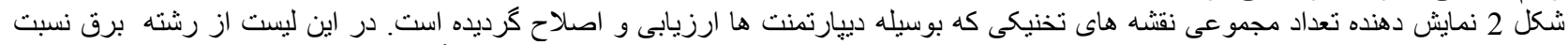

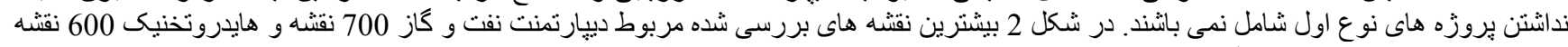

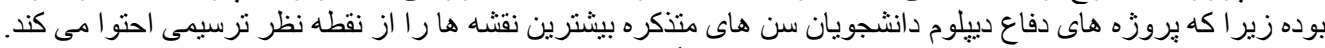

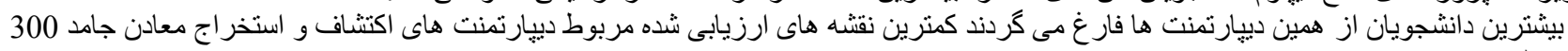

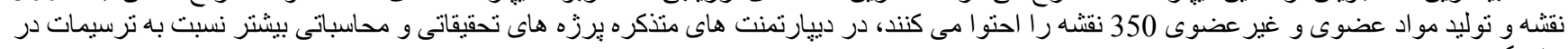
نظر كرفته مى شود مواد 


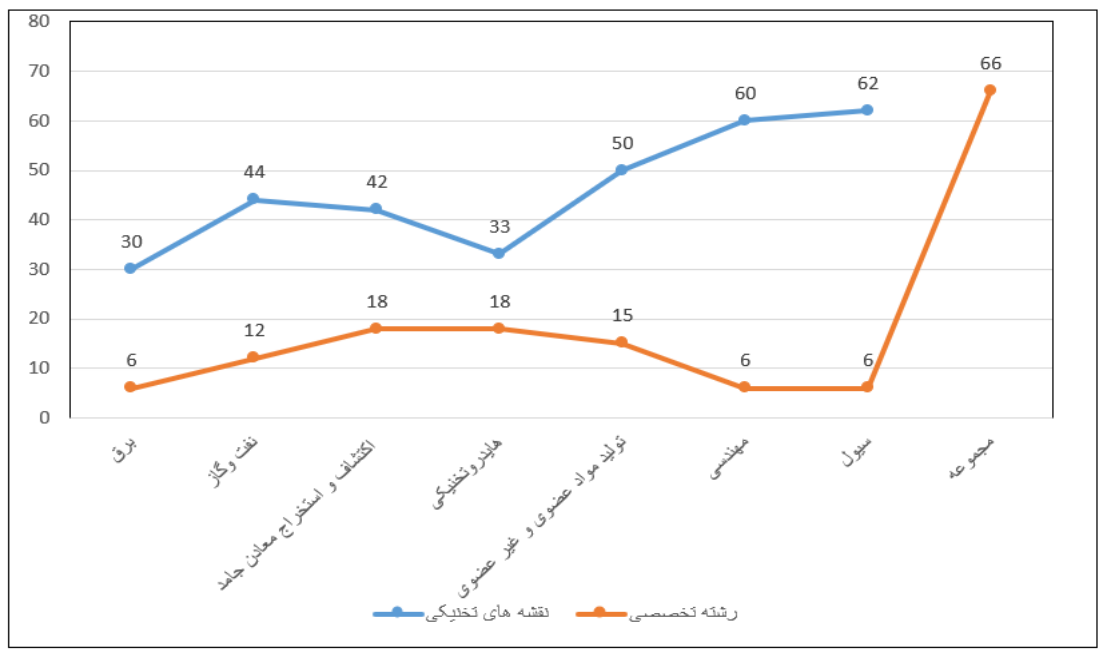

شكل 1. بِروزه هاى مطالعه شده در رشته هاى مختلف انجنيرى.

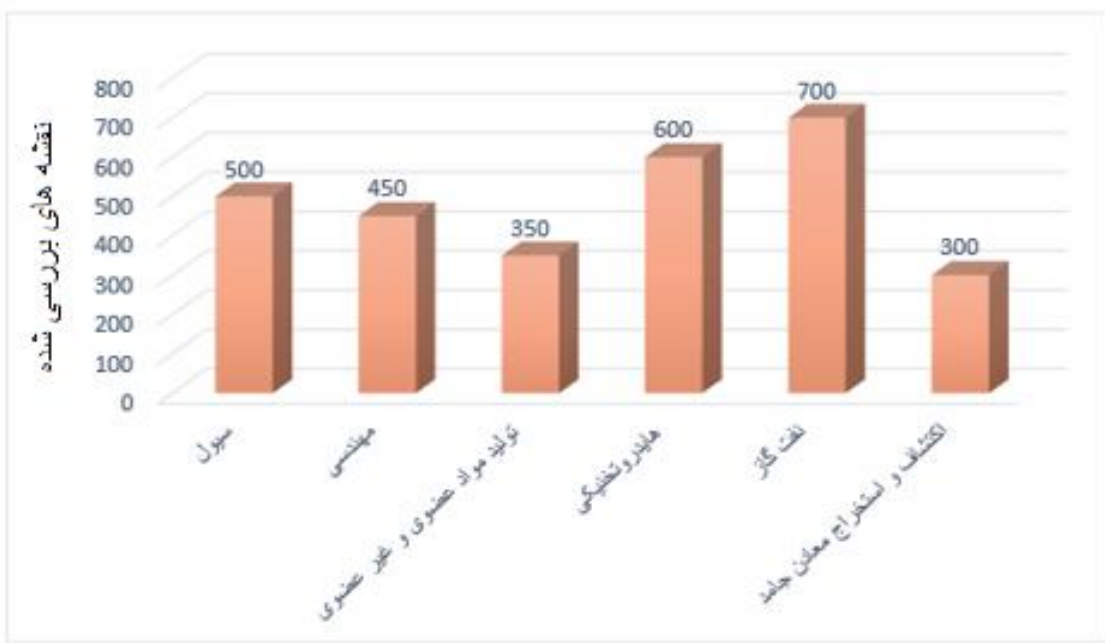

شكل 2. تعداد نقثه هاى رسم تخنيكى كه توسط اساتيد بررسى و اصلاح شده است.

2.3

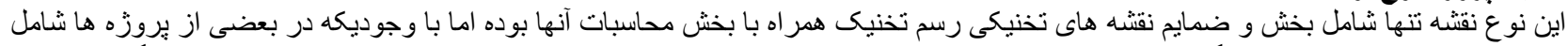

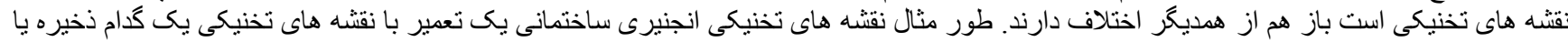

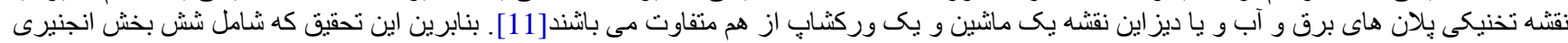

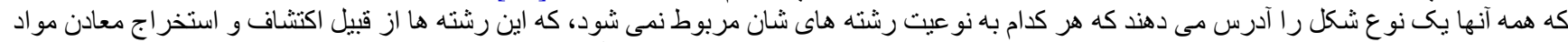

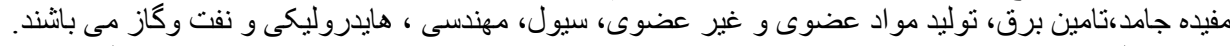

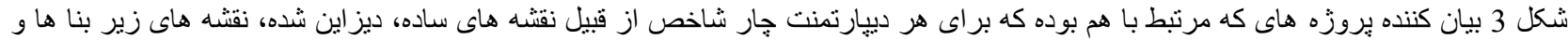

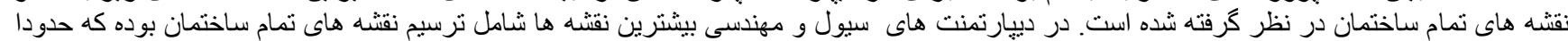

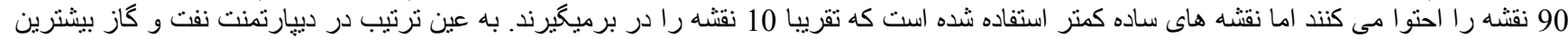

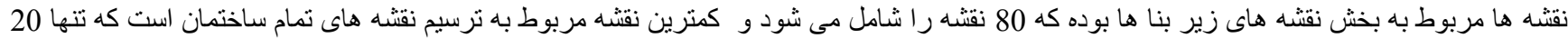

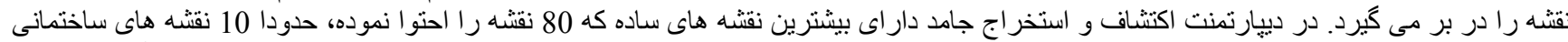

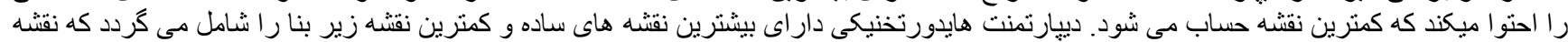

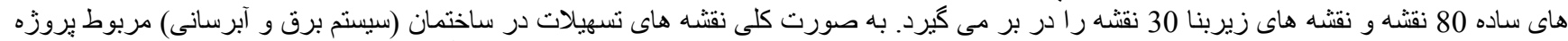

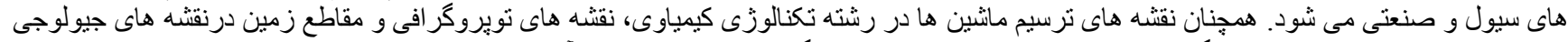

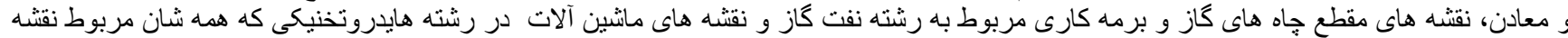
هاى مغلق انجنيرى مى شوند.

3.3

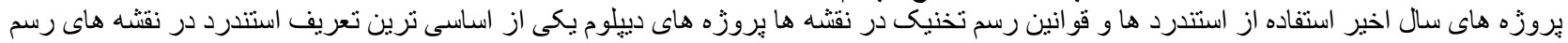

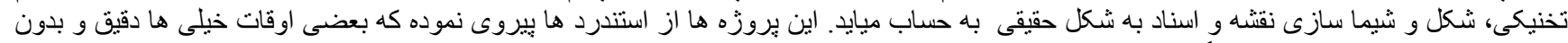

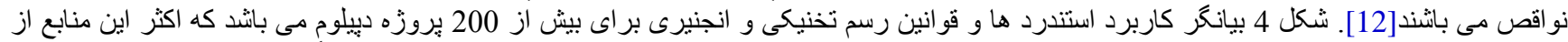

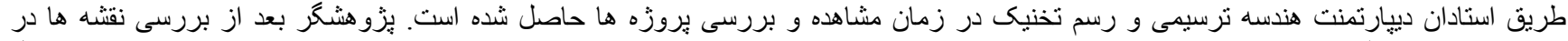

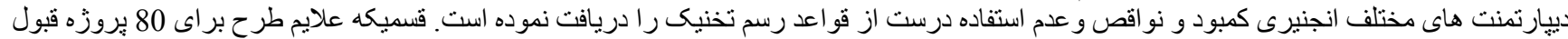

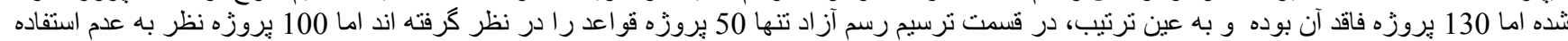

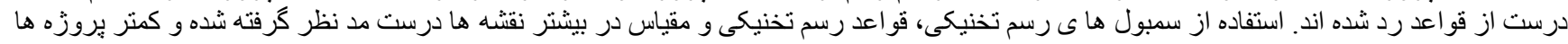




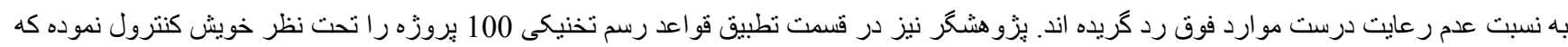

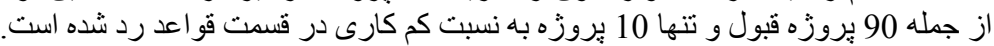

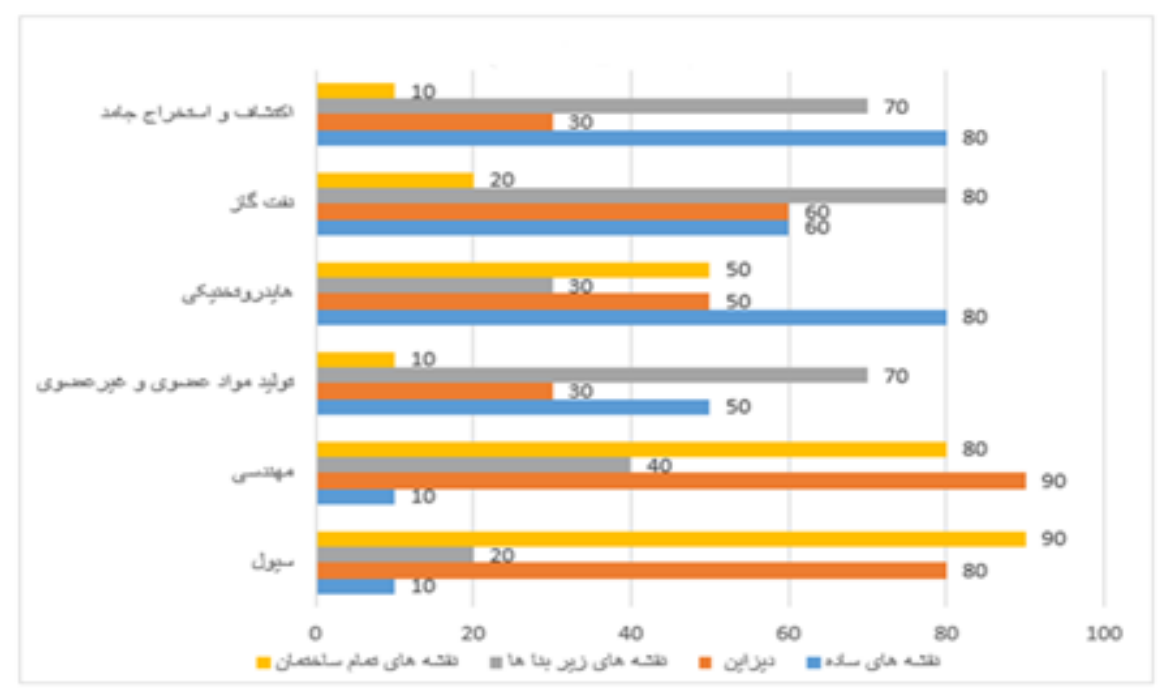

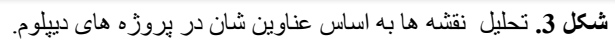

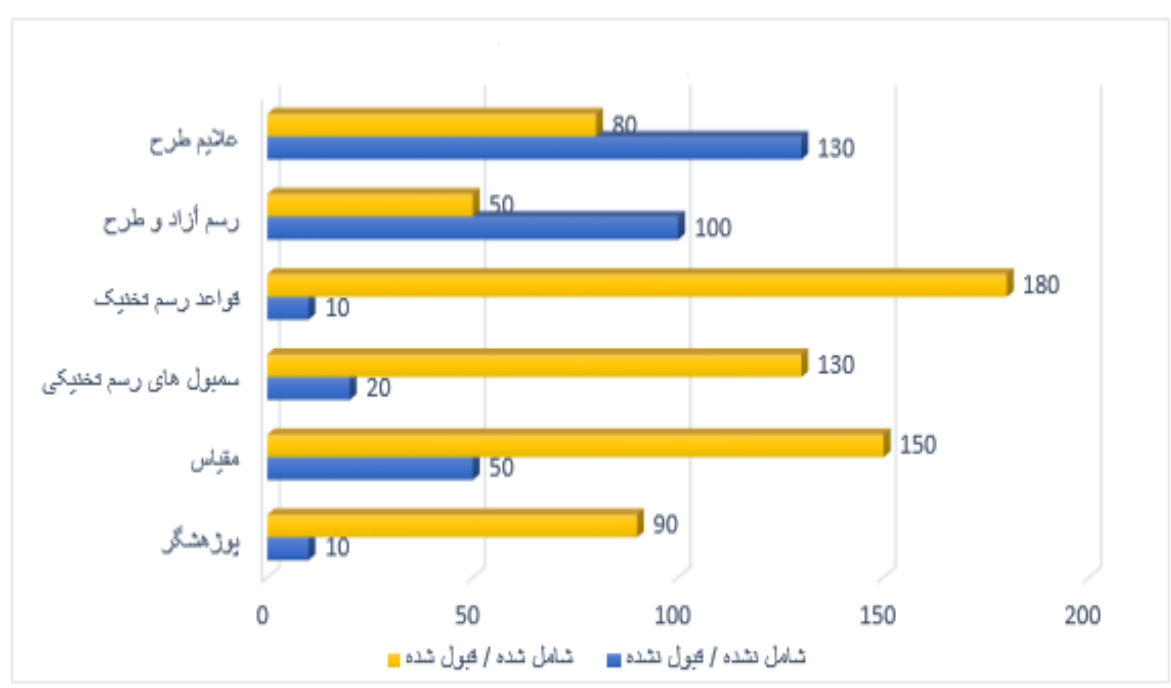

شكل 4. استفاده مشخصات داخل جوكات با اجزاى آن در بروزه ها و نقشه ها.

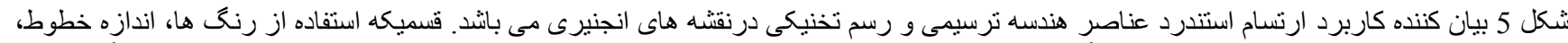

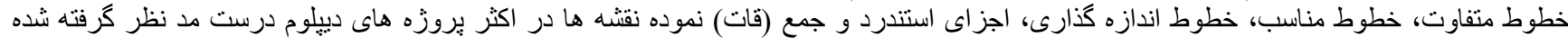

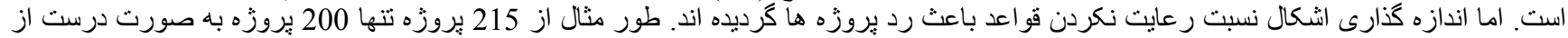

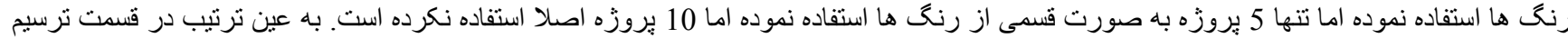

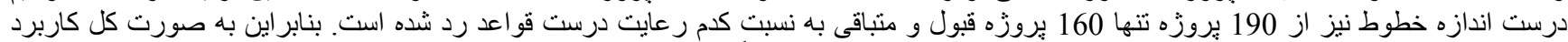

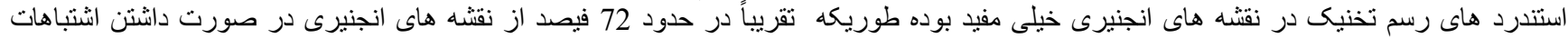

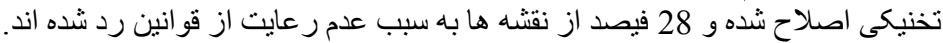

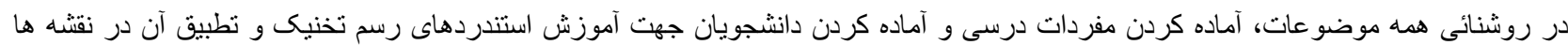

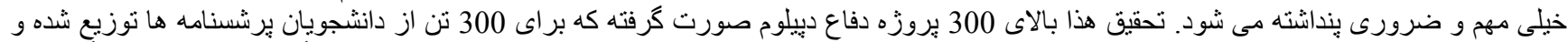

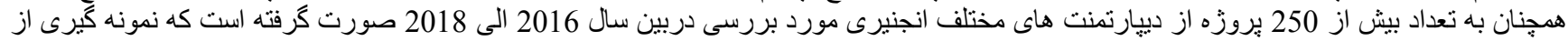

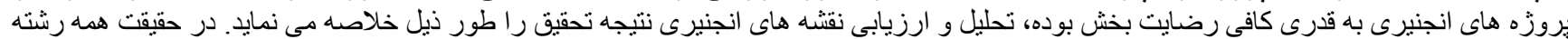

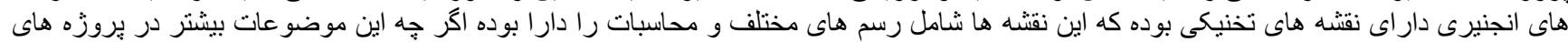

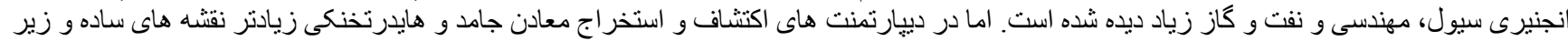

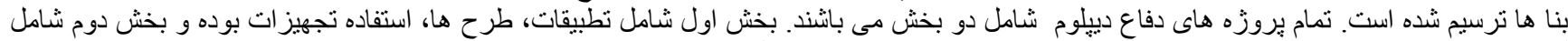

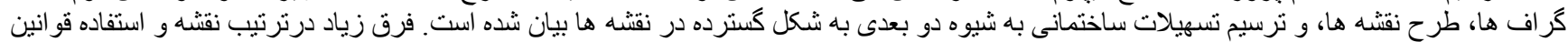

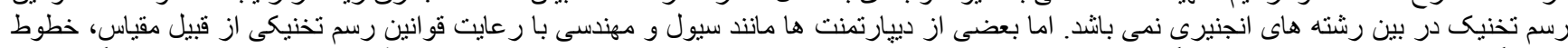

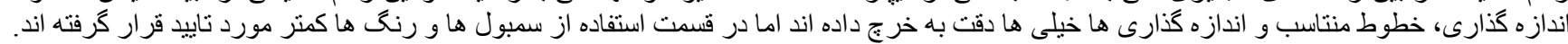

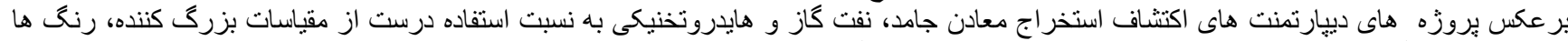

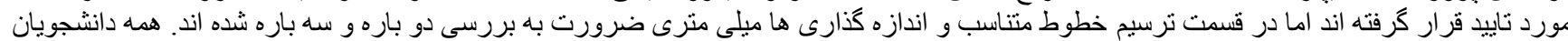

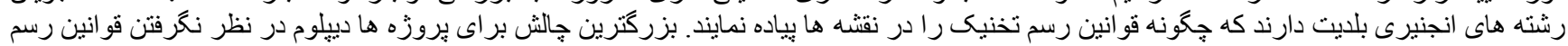




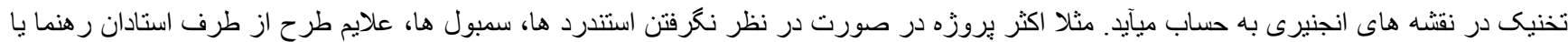

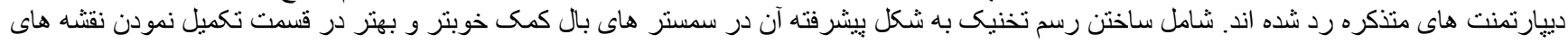
سال اخير مى نمايد.

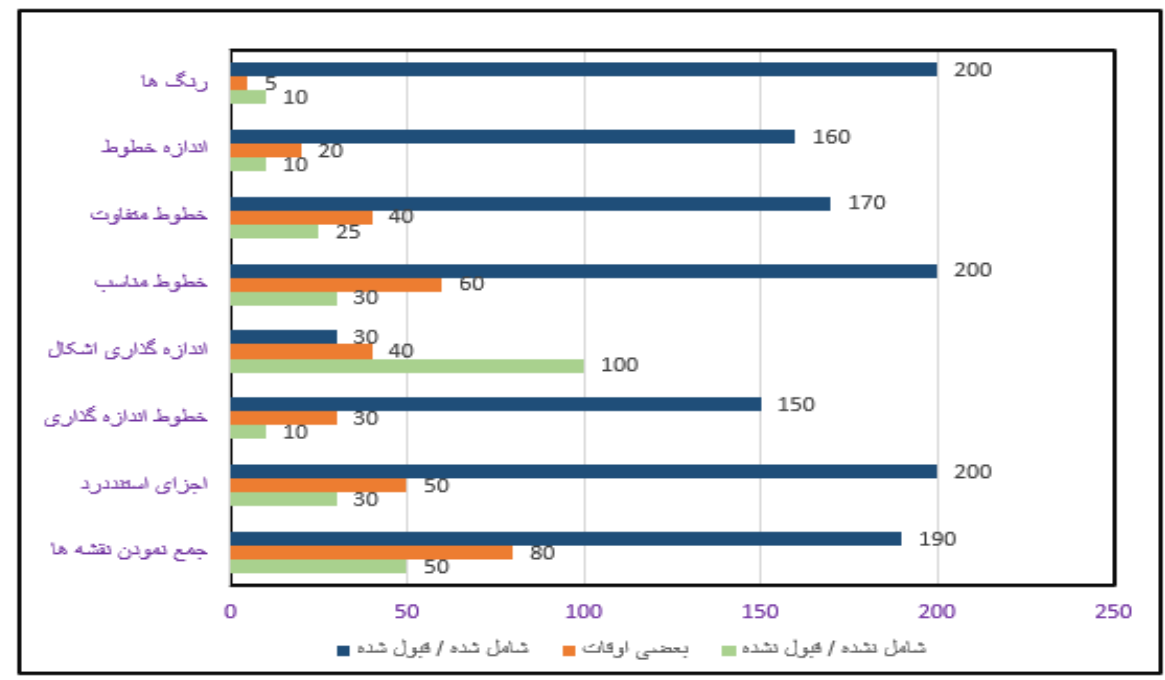

شكل 5. كاربرد استندرد ها در طرح بِروزْه هاو نقتشه هاى انجنيرى.

\section{References}

[1] J. Jawitt, S. Sahy, and R. Moore, "Management and assessment of final year projects in engineering," International Journal of Engineering Education, vol. 18, pp. 472-478, 2002.

[2] Jorjani, General building drawing - 2nd degree technical skills. Tehran: Danesh va Fan Publications (1397 AH) $816,1397$.

[3] S. Mohammadi, Farrokhzad, technical construction drawing. Tehran: Textbook Publishing Company (2012) 1-88, 2012.

[4] Durandish, Technical drawing and general building drawing. Tehran: Textbook Publishing Company (2012) $270,2012$.

[5] Zomarshidi, Technical drawing and cartography. Tehran: Jame Omran, Emerald Publisher (2016) $308,2016$.

[6] Arch, Technical drawing and drawing. Tehran: Textbook Publishing Company (2014) 179, 2014.

[7] Rezaei, Specialized technical drawing. Tehran: Gavayesh No (2012) 153, 2012.

[8] Mottaghipour, "Industrial cartography," ed Tehran: Afarang, 2013, pp. 4-20.

[9] G. Vitner and S. Rozenes, "Final -Year projects as a major element in the IE curriculum," European J. of Engineering Education, vol. 34, pp. 587-592, 2009. Available at: https://doi.org/10.1080/03043790903202975.

[10] A. Rafi, K. A. Samsudin, and A. Ismail, "On improving spatial ability through computer-mediated engineering drawing instruction," Educational Technology \& Society, vol. 9, pp. 149-159, 2006.

[11] C. J. Atman, D. Kilgore, and A. McKenna, "Characterizing design learning: A mixed-methods study of engineering designers' use of language," Journal of Engineering Education, vol. 97, pp. 309-326, 2008. Available at: https://doi.org/10.1002/j.21689830.2008.tb00981.x.

[12] S. K. Kataria, Engineering drawing geometrical, 1st ed.: Puna University, 2012. 